\title{
Surveillance of invasive Neisseria meningitidis with a serogroup Y update, Sweden 2010 to 2012
}

B Törös (nora-bianka.toros-vig@orebroll.se) ${ }^{1}$, S Thulin Hedberg ${ }^{1}$, S Jacobsson'1 ${ }^{1}$ H Fredlund ${ }^{1}$, P Olcén ${ }^{1}$, P Mölling ${ }^{1}$

1. National Reference Laboratory for Pathogenic Neisseria, Department of Laboratory Medicine, Clinical Microbiology/Molecular diagnostics R\&D, Örebro University Hospital, Örebro, Sweden

Törös B, Thulin Hedberg S, Jacobsson S, Fredlund H, Olcén P, Mölling P. Surveillance of invasive Neisseria meningitidis with a serogroup Y update, Sweden 2010 to 2012. Euro Surveill. 2014;19(42):pii=20940. Available online: http://www.eurosurveillance.org/ViewArticle.aspx?Articleld=20940

An increase of invasive meningococcal disease caused by Neisseria meningitidis serogroup $\mathrm{Y}$ has been noted in Sweden since 2005, and to a lower extent throughout Europe. The present study describes the epidemiology of invasive $N$. meningitidis isolates in Sweden in the period between 2010 and 2012, with a focus on serogroup Y. We also aimed to find an optimal molecular typing scheme for both surveillance and outbreak investigations. All invasive $N$. meningitidis isolates in Sweden during the study period $(n=208)$ were genetically characterised. Serogroup $Y$ predominated with $22 / 57,31 / 61$ and 44/90 of all invasive isolates (incidence $0.23,0.33$ and 0.46 per 100,000 population) in 2010, 2011 and 2012 respectively. In each of these years, 15/22, 22/31 and 19/44 of serogroup $Y$ isolates were genetically clonal (Y: $\mathrm{P}_{1.5}-2,10-1,36-2$ : $\mathrm{F}_{4}-1$ : ST-23(cc23), 'porB allele 3-36, fHbp allele 25 and penA allele 22). Our findings further support those of others that currently recommended FetA typing could be replaced by FHbp. Moreover, in line with a previous study that we conducted, the current results indicate that highly variable multilocus variable-number tandem repeat analysis (HV-MLVA) can be used as a firsthand rapid method for small outbreak investigations.

\section{Introduction}

Neisseria meningitidis (the meningococcus) is a Gramnegative diplococcus carried asymptomatically in the pharynx by approximately $10 \%$ of the population [1] It is also a potentially devastating pathogen causing meningitis and septicaemia. Invasive meningococcal disease (IMD) occurs mainly in sporadic cases but also as outbreaks and epidemics. Meningococcal populations are genetically and antigenically highly diverse [2] and vary greatly globally and over time, but the majority of IMD is caused by a limited number of clonal complexes, known as hyper-virulent lineages [3]. Therefore, detailed characterisation of circulating meningococcal strains is important in terms of vaccination policy decisions, outbreak management, as well as monitoring antibiotic susceptibility and vaccine coverage.
The polysaccharide capsule surrounding the bacterium defines the meningococcal serogroup. The capsule is an important virulence factor and IMD is mainly restricted to encapsulated meningococci belonging to serogroups A, B, C, W, X and Y. The capsule is also a polysaccharide vaccine component in available conjugate vaccines for serogroups $A, C, W$ and $Y$ [4]. The serogroup distribution is highly regional [5]. In Europe, the main circulating strains belong to serogroups $B$ and $C$ [6]. As previously described, serogroup $Y$ has increased in Sweden from 0.04 per 100,000 population in 2005 to 0.23 per 100,000 population in 2010 [7]. An emergence of serogroup $Y$ has also been noted in some other European countries, however, the highest relative proportions are found in Scandinavia $[8,9]$.

In addition to serogroup designation, it is currently recommended by the European Meningococcal Disease Society (EMGM) that meningococcal strains are designated by variable regions (VR) in the Porin A (PorA) and the Ferric enterobactin transport protein A (FetA) proteins as well as multilocus sequence typing (MLST) sequence type (ST) and clonal complex (CC) [10]. PorA and FetA are two surface antigens, which are recommended for rapid investigation of disease outbreaks. MLST, based on seven housekeeping genes, is ideal for studying population biology and evolution of the organism on a national and international level. For enhanced resolution, genotyping of a third surface antigen, Porin B (PorB), may also be performed [11]. Finally, further characterisation can be achieved with the penA gene encoding the penicillin-binding protein 2 (used in surveillance of penicillin susceptibility) [12] and $\mathrm{fHbp}$ encoding the serogroup $\mathrm{B}$ vaccine component Factor $\mathrm{H}$ binding protein $(\mathrm{FHbp})[13,14]$.

Another molecular method that has been proposed for an alternative typing paradigm, mainly suited for investigating localised outbreaks, is multilocus variable-number tandem repeat analysis (MLVA) [15-18]. MLVA is a polymerase chain reaction (PCR)-based technique, which uses the variability in the numbers of short tandem repeats to create DNA fingerprints used 


\section{FIGURE 1}

Incidence of invasive meningococcal disease caused by Neisseria meningitidis serogroups B, C, W and Y in Sweden, 2000-2012 $(\mathrm{n}=642)$

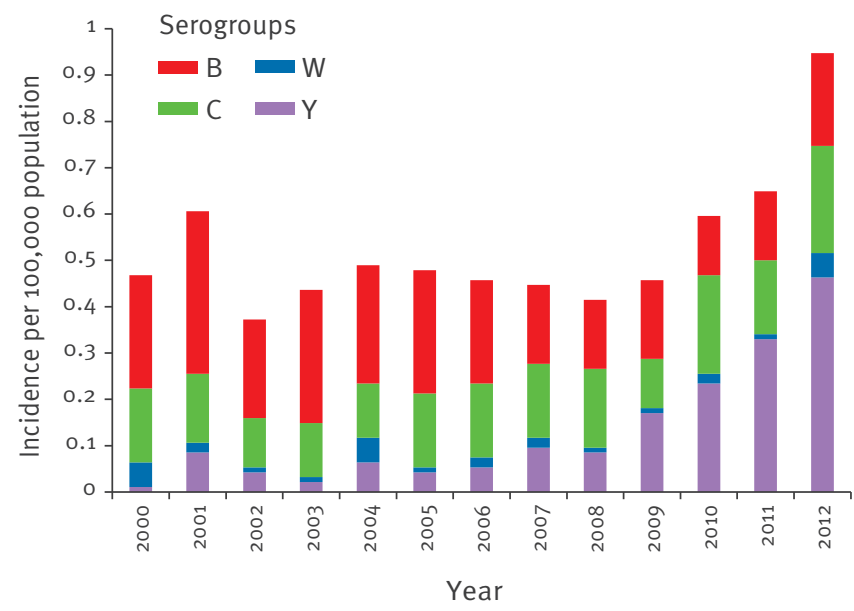

in epidemiological studies. The highly variable MLVA (HV-MLVA) developed by Schouls et al. [18] has shown high discriminatory capacity for serogroup $C$ isolates and has been considered suitable for outbreak identification [19].

The aims of the present study were to describe the current epidemiology of invasive $N$. meningitidis isolates including the dominating serogroup $Y$ in Sweden, and to find an optimal molecular typing scheme with appropriate resolution power for both surveillance and outbreak investigations.

\section{Methods}

\section{Bacterial isolates and phenotypic}

characterisation

In Sweden, all invasive cases of meningococcal disease according to the European Union case definition are mandatorily reported by clinicians to the Swedish Institute for Infectious Disease Control (SMI) [20]. The corresponding isolates are sent to the Public Health Agency of Sweden, where they are routinely cultured on chocolate agar at $37^{\circ} \mathrm{C}$ with $5 \% \mathrm{CO}_{2}$ overnight and subsequently serogrouped by co-agglutination [21]. Further genosubtyping (PorA typing) is then conducted as previously described [22] and antibiotic susceptibility determined using the Epsilometer (E)test method (bioMérieux, Marcy l'Etoile, France). Basic epidemiological data (age, sex, area of residence, clinical site of isolation and date of sample collection) are gathered for all isolates from cases. This study included all invasive $N$. meningitidis isolates in Sweden between 2010 and 2012. The serogroup B strain $M C_{5} 8$ [23] was included in all analyses as a reference.

\section{Nucleic acid extraction}

The DNA used for sequence-based typing methods and MLVA was extracted with a NorDiag Bullet instrument (DiaSorin, Dublin, Ireland). For the automatic extraction, 20 colonies from each cultured organism were suspended in $2 \mathrm{ml} \mathrm{NaCl}(0.85 \%)$ and $100 \mu \mathrm{l}$ of this solution were subsequently processed with the NorDiag Bullet with the Bullet BUGS'n BEADS kit according to the manufacturer's recommendation (DiaSorin). All DNA preparations were stored at $4^{\circ} \mathrm{C}$ prior to the PCR.

\section{FIGURE 2}

Distribution of the genetically defined predominant Neisseria meningitidis clone YI with different sulfamethoxazole susceptibilities, the second and third most common serogroup Y clones (YII and YIII) and all other invasive N. meningitidis serogroup Y isolates in Sweden, 2000-2012 ( $n=163)$

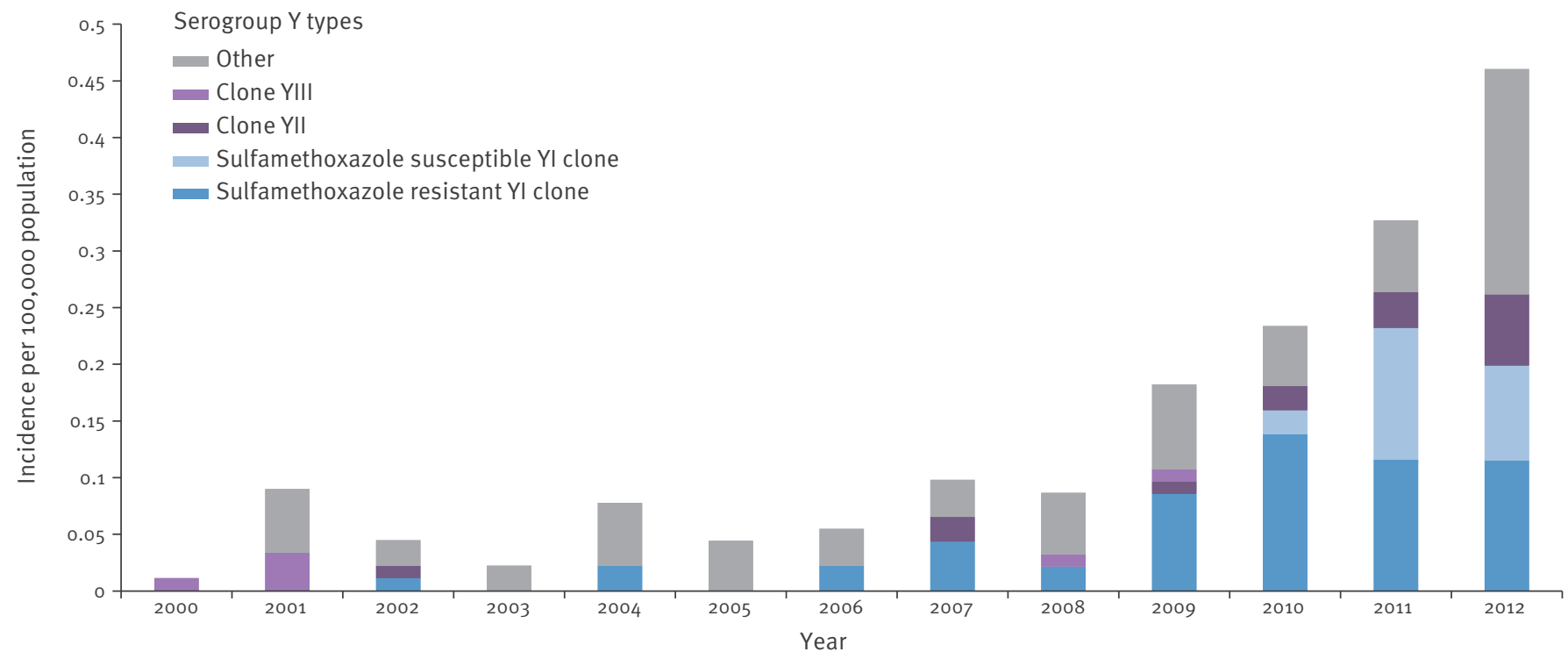

Data for this Figure originate both from this study and a previous one [7]. 


\section{FIGURE 3}

Serogroup distribution in the most frequent PorA, FetA and $\mathrm{fH} b \mathrm{p}$ variable regions (VRs) or alleles of invasive Neisseria meningitidis isolates in Sweden, 2010-2012

A. Serogroup distribution by PorA VR

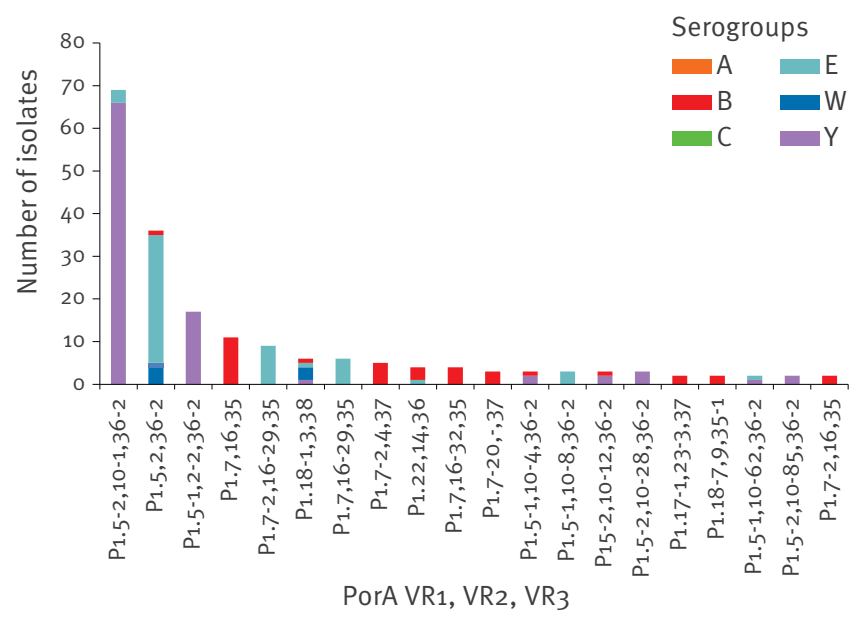

B. Serogroup distribution by FetA VR

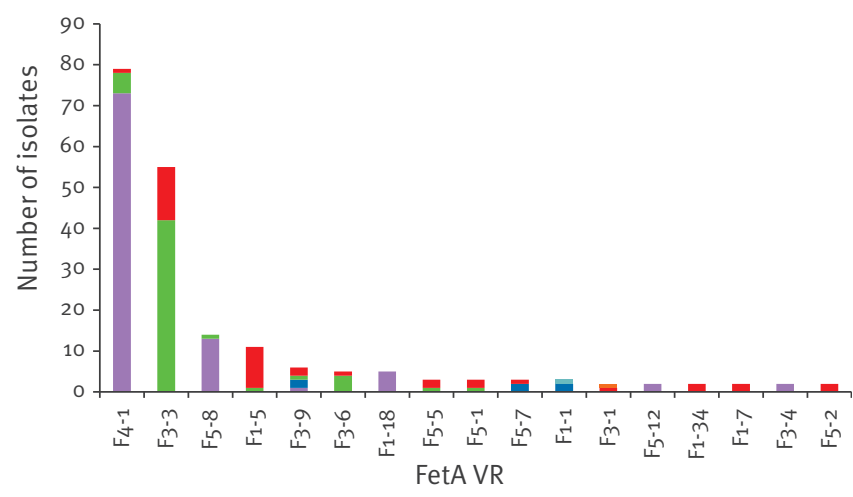

C. Serogroup distribution by $f H b p$ allele

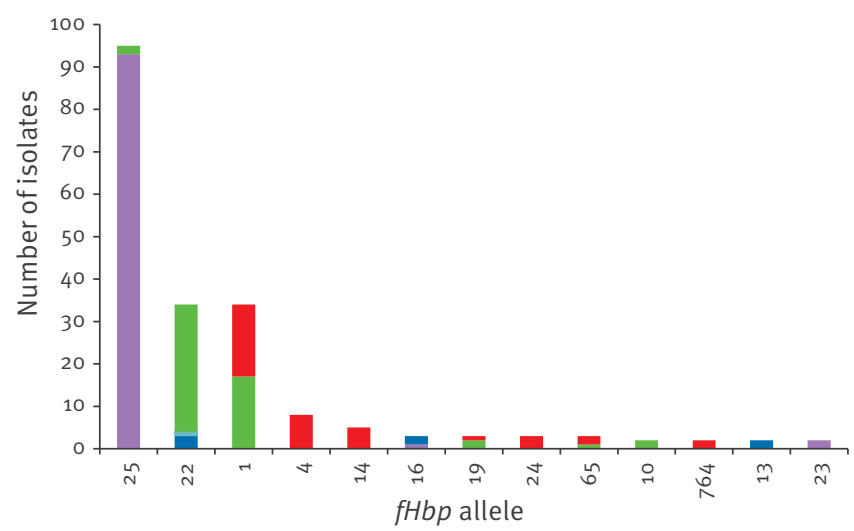

FetA: Ferric enterobactin transport protein A; PorA: Porin A. Only the types represented by at least two isolates are displayed.
Polymerase chain reaction and DNA sequencing

The MLST genes: $a b c Z, a d k, a r o E, f u m C, g d h, p d h C$ and $p g m$ together with $f e t A, f H b p$, porB and penA were amplified and sequenced as previously described $[7,19]$. In short, the PCR was performed using a Rotor-Gene Q real-time PCR system (Qiagen, Hilden, Germany) and detected with SYBR green I. The nucleotide sequences were determined by capillary electrophoresis using an ABI PRISM 3130xl Genetic Analyzer (Applied Biosystems, Foster City, CA, USA) using BigDye Terminator v3.1 (Applied Biosystems, Warrington, UK). The sequence alignments for the MLST genes were assembled using the Bionumerics software version 6.6 (Applied Maths, Sint-Martens-Latem, Belgium, http:// www.applied-maths.com/bionumerics) and all other genes were assembled using the ChromasPro software version 1.33 (Technelysium Pty Ltd, Brisbane, Australia, http://technelysium.com.au/). The different sequences were assigned allele numbers using the $N$. meningitidis sequence query database [24].

\section{Multilocus variable-number tandem repeat analysis}

The HV-MLVA with four highly variable variable-number tandem repeat (VNTR) loci (VNTR4-4, VNTR9-2, VNTR4-2, and VNTR4-3) was performed as previously described by Törös et al. [19] with the primers from Schouls et al. [18]. In short, the PCR was performed on an Applied Biosystems 9700 or 2720 PCR machine and the fragments were separated on an ABI PRISM $3130 x$ l Genetic Analyzer using the GeneScan LIZ 500 size standard and GeneScan module with filter set G5 (Applied Biosystems). The sizing of the fragments was performed with the GeneMapper software v4.0 (Applied Biosystems). All isolates were run in duplicates in separate runs.

\section{Data analysis}

The ability of each method to discriminate between strains was evaluated on the basis of their discrimination index, using Simpson's index of diversity (ID) [25]. The ID determines the probability that two randomly picked strains are allocated to different types. Confidence intervals (Cl) of 95\% were calculated [26]. Cluster analysis of the MLST data was performed using a categorical coefficient and displayed in a minimum spanning tree (MST) created with the Bionumerics software version 7.1, with the priority rule of first linking types which have the highest number of single-locus variants. For the HV-MLVA, a dendrogram was generated using a categorical coefficient and unweighted pair group method with arithmetic average (UPGMA).

\section{Results}

\section{Epidemiology}

A total of 208 invasive $N$. meningitidis isolates were characterised during the study period, including 57 in 2010, 61 in 2011 and 90 in 2012. The isolates originated from clinical specimens of cerebrospinal fluid $(n=44)$, 
Minimum spanning tree from multilocus sequence typing profile data for Neisseria meningitidis isolates in Sweden, 2010$2012(n=208)$

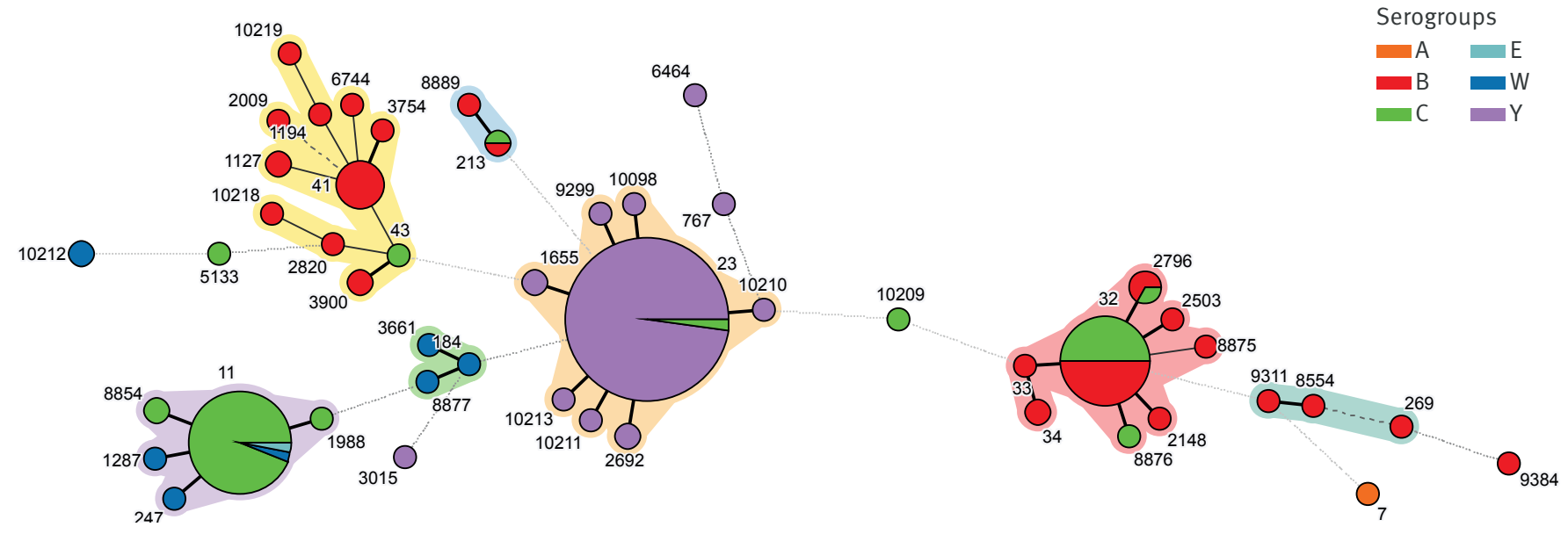

Each circle represents a sequence type (ST), and the serogroup distribution in each ST is represented by the respective colour.

Lines connecting the STs that are thick and solid, thin and solid, dashed and dotted denote 1, 2, 3 and 4 or more loci differences, respectively. Halos surrounding the circles denote different clonal complexes. Purple: clonal complex ST-11; yellow: clonal complex ST-41/44; green: clonal complex ST-22; blue: clonal complex ST-213; peach: clonal complex ST-23; pink: clonal complex ST-32; turquoise: clonal complex ST-269.

tissue $(n=1)$, joint fluid $(n=4)$ and blood $(n=159)$. Among all isolates, 97 belonged to serogroup $\mathrm{Y}, 57$ to serogroup $C, 44$ to serogroup $B$, eight to serogroup $W$, one to serogroup $A$ and one to serogroup $E$. The annual incidences of all meningococcal serogroups in Sweden from 2000 to 2012 are described in Figure 1. The figure also shows that the total incidence of IMD in Sweden has increased prominently in 2012, from approximately 0.6 per 100,000 population in 2010 and 2011 to 0.95 per 100,000 population in 2012. The predominant serogroup in the period between 2010 and 2012 was serogroup $\mathrm{Y}$, which accounted for $22 / 57$ (39\%, incidence 0.23 per 100,000 population), 31/61 (51\%, incidence 0.33 per 100,000 population) and $44 / 90$ (49\%, incidence 0.46 per 100,000 population) of all invasive isolates in Sweden in 2010, 2011 and 2012, respectively.

The clonal pattern of the invasive serogroup $\mathrm{Y}$ isolates is presented in Figure 2. Of all serogroup $Y$ isolates, $15 / 22(68 \%)$ in 2010, 22/31 (71\%) in 2011 and 19/44 (43\%) in 2012 were genetically identical to clone $\mathrm{YI}$ as previously described by Thulin Hedberg et al. [7] (Y: P1.5-2,10-1,36-2: F4-1: ST-23(cc23), 'porB allele 3-36, fHbp allele 25 and penA allele 22). However, a sulfamethoxazole susceptible variant of the clone seems to have appeared in 2010, which in 2011 corresponded to $11 / 22(50 \%)$ of the predominant clone, and $8 / 19$ (42\%) in 2012. As seen in Figure 2, the previously described clone YII [7] (sulfamethoxazole susceptible, with type Y: P.5-1,2-2,36-2, F5-8, ST 23(cc23), "porB allele $2-55, f H b p$ allele 25 and penA allele 22) is still the second most frequent serogroup $\mathrm{Y}$ clone corresponding to $3 / 22$ (14\%) in 2010, 3/31 (10\%) in 2011 and $6 / 44$ (14\%) in 2012.
The current clonal pattern among serogroup $\mathrm{Y}$ isolates is further outlined in Figure 3 and 4 where the PorA types $\mathrm{P}_{1.5}-2,10-1,36-2$ and $\mathrm{P}_{1} .5-1,2-2,36-2$, FetA VR $\mathrm{F}_{4-1}$ and 5-8, fHbp allele 25 and ST-23 are the most frequent. Furthermore, 29/57 (51\%) of all serogroup

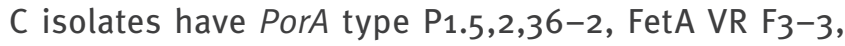
fHbp allele 22 and ST-11 (Figures 3 and 4).

The age distribution of patients with IMD during 2010 to 2012 is shown in Figure 5. Serogroup B was most common among children (median age: 19 years; inter quartile range (IQR): 7-33), serogroup $C$ among young adults (median age: 20 years; IQR: 17-59) and serogroup $\mathrm{Y}$ among older adults (median age: 64 years; IQR: 37-77). The figure shows that among IMD caused by serogroup $\mathrm{Y}$ during this time period, the genetically defined predominant clone $\mathrm{YI}$ and clone $\mathrm{YII}$ are mainly common among an older age group compared to the other serogroup $Y$ isolates. The mortality rate among patients with IMD caused by serogroup Y from 2010 to 2012 was $6 / 97$ (6\%; $95 \% \mathrm{Cl}: 1.4-11 \%$ ) compared to the total mortality rate due to all serogroups combined during the same time period which was 18/208 (9\%; 95\% $\mathrm{Cl}: 5-12 \%])$. The IMD mortality rate in Sweden during 2000 to 2009 was $67 / 569$ (12\%; 95\% Cl: 9-14\%).

\section{Resolving power of molecular typing methods used for surveillance}

The discriminative ability of a MLST and the porA, por $B$, fet $A, f H b p$ and $p e n A$ genes shows that as single targets PorA VR 1, 2, and 3 had the highest Simpson's ID (0.849; 95\% Cl: 0.811-0.886) and serogroup had the lowest (0.664; 95\% Cl: 0.628-0.700]). Among combinations of four, on the basis of serogroup, porA and MLST, this combination including fet $A$ had the highest 
Age distribution of patients with invasive meningococcal disease caused by Neisseria meningitidis serogroups B, C, Y, the genetically defined predominant serogroup Y clone YI and the second most common serogroup Y clone (YII) in Sweden, $2010-2012(n=208)$

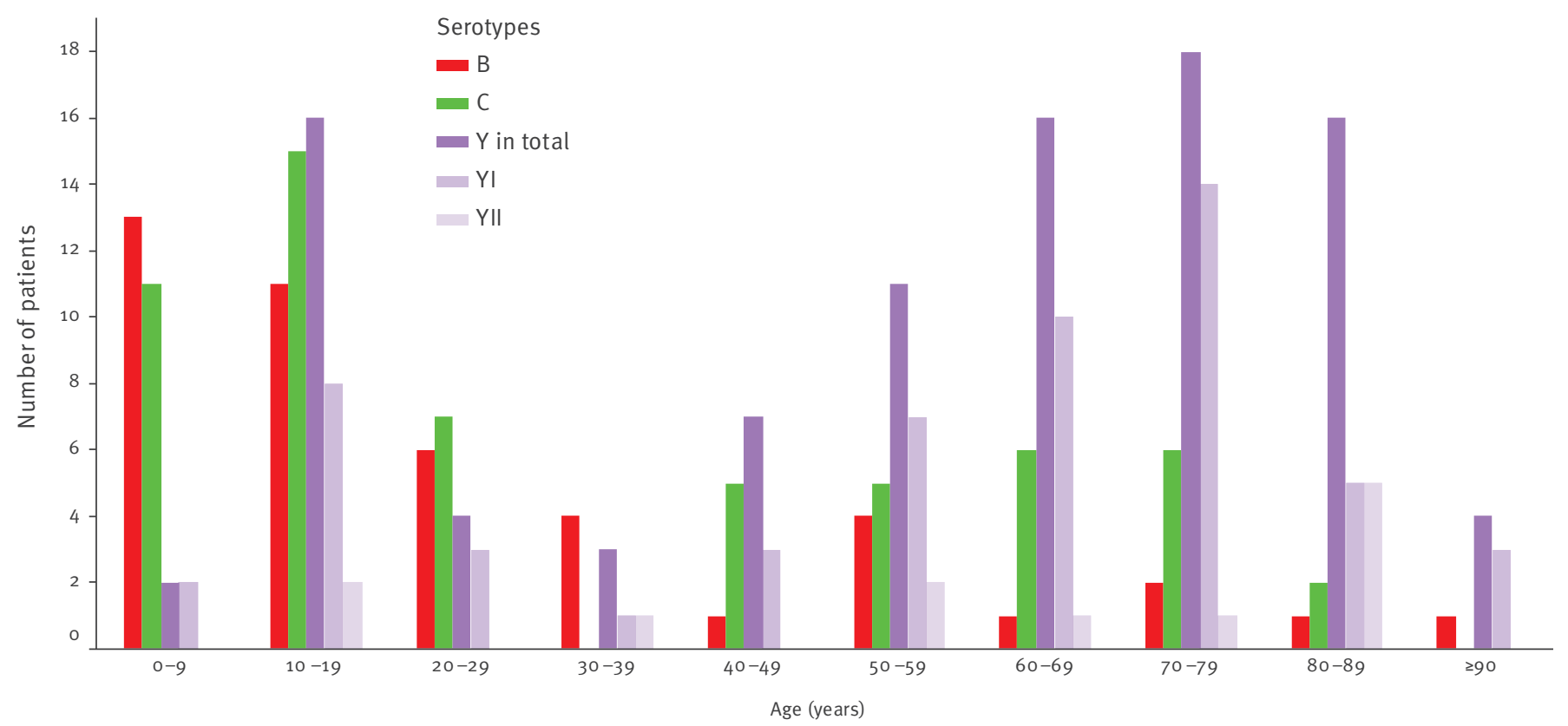

ID (0.956; 95\% Cl: $0.940-0.972)$ and serogroup, porA and MLST including fHbp had the lowest (0.950; $95 \%$ Cl: 0.933-0.967).

\section{Outbreak investigations}

A spatiotemporal association was defined as isolates of the same serogroup, collected in the same central county within a timeframe of one month. Of the 208 N. meningitidis invasive isolates from 2010 to 2012, 35 isolates were spatiotemporally associated in 16 different clusters and represented potential outbreaks. There were 13 clusters of two isolates and three clusters of three isolates. Eight of the spatiotemporal clusters (cluster nr 1-8 in Figure 6) did not share a common PorA type within the clusters and the cases had not been in direct contact with each other and therefore there were no further outbreak investigations. In spatiotemporal clusters $\mathrm{nr} 10-13$, the isolates were identical within each cluster regarding serogroup and all 12 target genes (Figure 6), but no connection between cases was found and the isolates were separated by HV-MLVA. Within each of the three spatiotemporal serogroup $B$ clusters 14-16 (Figure 6) the isolates were identical regarding all 12 sequenced genes (the isolates in cluster 14 all had one insertion in aroE allele 9 but were still regarded as belonging to ST-41). In addition, the isolates were clustered together in the HV-MLVA when MLVA types did not differ in more than one VNTR locus (single-locus variant, SLV). However, spatiotemporal cluster 14 and 16 were the only spatiotemporal clusters which had confirmed connections between cases. The HV-MLVA results from all 208 isolates showed another seven HV-MLVA clusters comprising fifteen isolates in total (if SLVs were allowed), of which four did have identical genetic profiles, but none of them shared a spatiotemporal link and were therefore not included in Figure 6.

\section{Discussion}

This study aimed to describe the epidemiology of invasive N. meningitidis isolates in Sweden between 2010 and 2012, specifically the dominating serogroup $Y$, and to identify an optimal molecular typing scheme with appropriate resolution power for both surveillance and outbreak investigations in low-endemic areas. Although whole genome sequencing is becoming more cost beneficial than traditional Sanger sequencing per isolate, optimal molecular typing schemes will still be important when fast results are needed, and for smaller laboratories that lack the need of a next generation sequencer. All isolates causing IMD in Sweden during 2010, 2011 and 2012 were characterised by capsular group, MLST, sequencing of the $\operatorname{por} A$, fet $A$, por $B$, penA, fHbp genes and a MLVA using four highly variable loci.

The epidemiology in Sweden has changed most notably with an increase of IMD between 2010 and 2012. IMD caused by serogroup $C$ has declined slightly from 2010 to 2011 and 2012, and serogroup B somewhat increased (Figure 1). The genetic characterisation of circulating $N$. meningitidis causing IMD shows that serogroup $Y$ was the most prevalent in Sweden, and the previously genetically described predominant clone $\mathrm{YI}$ [7] was still dominating among serogroup $Y$ strains. However, the overall IMD incidence is still low and although a vaccine against serogroup $\mathrm{Y}$ is available, it is probably not 
Dendrogram of invasive Neisseria meningitidis isolates generated from a highly variable multilocus variable-number tandem repeat analysis, Sweden, 2010-2012 ( $\mathrm{n}=35$ isolates)

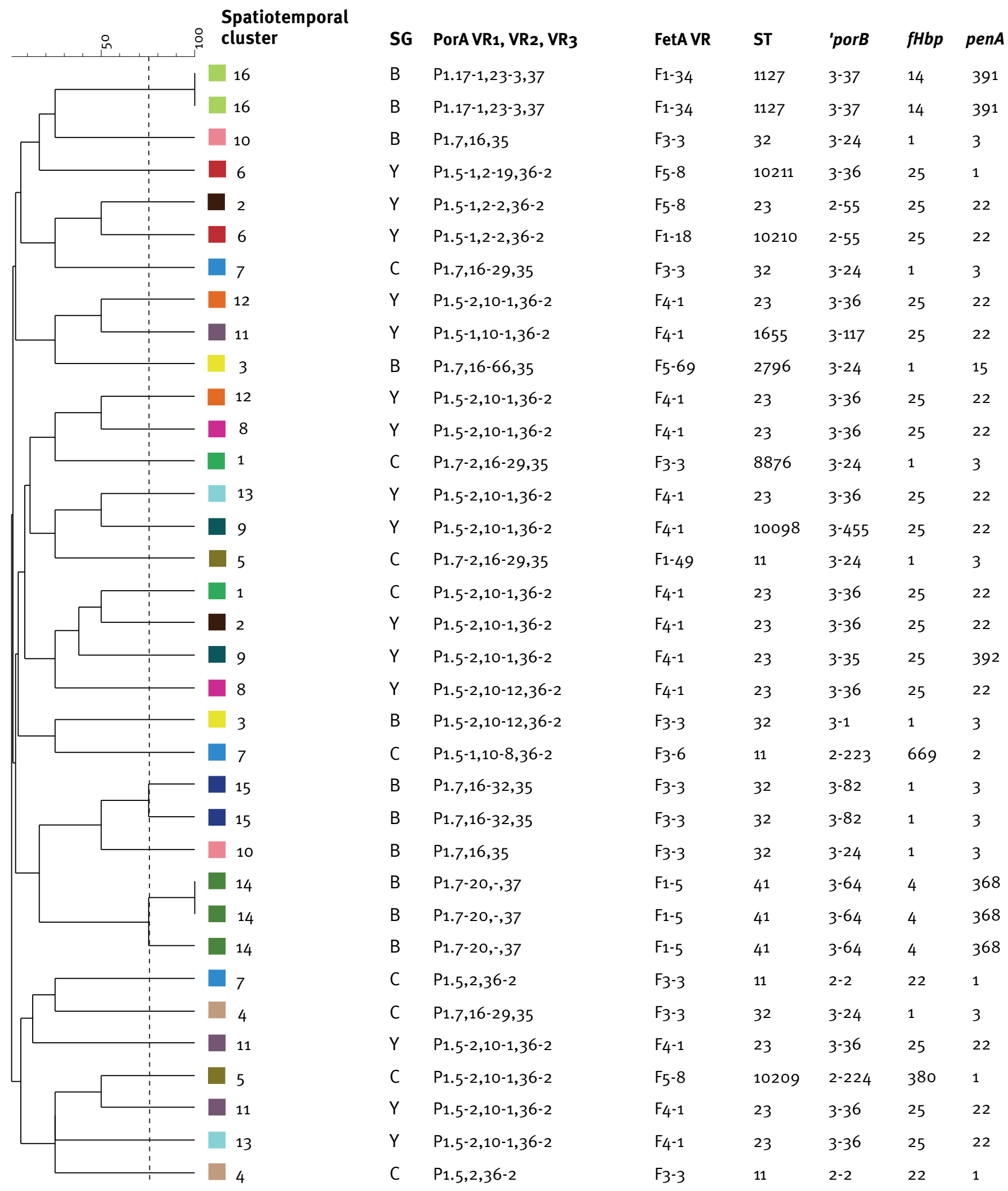

FetA: Ferric enterobactin transport protein A; PorA: Porin A; SG: serogroup; ST: sequence type; VR: variable region.

A similarity line has been drawn at 75\% (maximum one single-locus variant). Only spatiotemporally associated isolates (Sweden, 2010-2012) are included and have been designated a spatiotemporal cluster number 1-16. Additional information about serogroup and allele or VR from sequencing the porA, fet $A$, "porB (partial fragment), $\mathrm{fH} b \mathrm{p}$ and penA genes and the ST from the multilocus sequence typing is provided for each strain. 
justifiable to change the vaccine policy in Sweden which currently does not include any vaccines against IMD in the general vaccination programme. Moreover, a shift of sulfamethoxazole susceptibility has occurred sometime around 2010 where isolates otherwise identical to the predominant serogroup $Y$ clone instead are susceptible to sulfamethoxazole. Sequencing of the folP gene in a representative collection of the serogroup $Y$ isolates in this study has not shown any of the mutations previously associated with sulphonamide resistance (data not shown) $[27,28]$. Investigating larger parts of the genome, including differences in expression, can possibly elucidate the mechanisms responsible for this sulfamethoxazole susceptibility shift. Like serogroup $\mathrm{Y}$, serogroup $\mathrm{C}$ also presented a fairly clonal pattern whereas serogroup $B$ isolates were rather genetically heterogeneous (Figure 3 and 4).

The age distribution of patients with IMD in Sweden during 2010 to 2012 regarding serogroups $B$ and $C$ is similar to the age incidence pattern during the period from 1995 to 2009 where serogroup B is most common among patients under 10 years of age and serogroup $C$ is most common in the age group including 10 to 19 year-olds (data not shown). The mean age among patients with IMD caused by serogroup $Y$ in 2010 to 2012, 58.9 years, has somewhat increased compared to the mean age of 54.5 years for this serogroup in 2000 to 2009 (data not shown). A considerably lower average age of serogroup Y patients in 2011 has been reported in Denmark (26 years), France (20 years), Italy (26.9 years), Portugal (15.5 years) and Spain (31 years) [9]. Further studies need to be performed to give a clearer picture as to whether the virulence or transmissibility is increased in the predominant serogroup Y clone. Moreover, the mortality rate for IMD has somewhat decreased during 2010 to 2012 compared to the mortality rate during 2000 to 2009 , however this was not statistically significant. The mortality rate among serogroup $Y$ cases has also decreased slightly between the periods 2000 to 2010 and 2010 to 2012, however the difference was again not statistically significant. The decrease may be partly due to the recent small decrease in median age among patients with IMD caused by meningococci belonging to this serogroup.

The resolving power of a molecular typing method is recommended to have a discrimination level of at least 0.90 [25]. Serogroup:porA:fetA typing has previously shown a value of 0.963 [29] which is similar to the discrimination index achieved in this study $(0.952 ; 95 \%$ $\mathrm{Cl}$ : $0.935-0.969)$ with the same typing targets (data not shown). Although serogroup:porA:fetA:ST had the highest index of diversity achieved in this study, our results show that replacing the $f e t A$ gene with $f H b p$ only reduces the discrimination ability by 0.006 (no statistical significant difference). Concurrently, Lucidarme et al. [30] investigated the comparability between $f e t A$ and $f H b p$ in terms of diversity, after it had been recommended in England and Wales to additionally incorporate $\mathrm{fHbp}$ for routine genotypic surveillance.
Their study (on 613 invasive isolates) actually showed that $\mathrm{fH} b \mathrm{p}$ had significantly (non-overlapping $95 \% \mathrm{Cls}$ ) better resolving power than $f e t A$. These findings indicate that the level of discrimination gained from $\mathrm{FHbp}$ is partially complementary to that of fetA. This could strengthen the argument that considering labour and cost, and with regard of the new serogroup $B$ vaccines, it would be beneficial to substitute the current routine marker $f e t A$ with $f H b p$.

In terms of outbreak investigations, HV-MLVA detected three small clusters with spatiotemporal connections and identical genetic profiles (spatiotemporal clusters $14-16$ in Figure 6), which further supports that these strains were truly involved in small outbreaks. Although no connection could be confirmed between cases in spatiotemporal cluster 15 , the cases were both of similar age and from the same county and thus the cases could still have been related.

Fifteen isolates in the present study were clustered in seven HV-MLVA clusters without having a spatiotemporal connection (data not shown). However, without a spatiotemporal connection, these would normally never have been subjected to a HV-MLVA analysis. Moreover, the results of the HV-MLVA suggest that, after identifying the capsular group and receiving clinical data, it could have been sufficient to perform only the HV-MLVA to get indications of potential outbreaks. In an outbreak situation where time is of high importance, not having to await the results of PorA typing or whole genome sequencing for the subsequent HV-MLVA analysis would be beneficial.

We have previously compared HV-MLVA to rep-PCR with the Diversilab system and DNA sequencing on invasive serogroup $C$ isolates which showed that HV-MLVA helped strengthen all of the spatiotemporal linkages [19]. The use of MLVA to trace transmission has been deemed questionable due to the low stability of VNTRs. Transmission-dependent variation of tandem repeats in meningococci has been investigated by Elias et al. [31] in a study using four highly variable VNTR loci together with another eight standard MLVA loci. The observed overall variation was considerably smaller than predicted and the method was considered most useful for outbreaks containing few transmissions. Considering this, HV-MLVA could be valuable in low-endemic areas such as Sweden where outbreaks are fairly rare and connected cases usually only consists of no more than three contacts, as shown by our results. A fast detailed typing of spatiotemporally linked cases which can separate outbreaks from sporadic cases is important to inform public health measures to control IMD, such as deciding whether or not to offer prophylaxis in the form of antibiotics or vaccines.

In summary, serogroup $Y$ was found to be the most prevalent serogroup in Sweden, and the previously genetically described predominant clone YI [7] (Y: P1.52,10-1,36-2: F4-1: ST-23(cc23), 'porB allele 3-36, 
fHbp allele 25 and penA allele 22) is still dominating. However, a sulfamethoxazole susceptibility subvariant of the clone appears to have emerged in 2010, and in 2011 represented $11 / 22$ (50\%) of the genetically defined predominant clone $\mathrm{YI}$ isolates. Our study supports previous studies that suggests that the FetA typing could be replaced by $\mathrm{FHbp}$ in the recommended designation (serogroup:porA:fetA:ST(CC)), which may be more suitable in the current vaccine era. Furthermore, this study including the additional serogroups $A, B, E, W$ and $Y$, strengthens previous results on serogroup $C$ isolates [19] suggesting that HV-MLVA is a first-hand rapid method for investigating outbreaks with few transmission events, where isolates have an identical serogroup and a common spatiotemporal connection.

\section{Acknowledgements}

This work was funded by grants from the Örebro County Council Research Committee and the Foundation for Medical Research at Örebro University Hospital, Örebro, Sweden.

\section{Conflict of interest}

None declared.

\section{Author's contributions}

Bianca Törös, Sara Thulin Hedberg and Paula Mölling were mainly responsible for the design and supervision of the study. Bianca Törös and Susanne Jacobsson performed the laboratory work. Bianca Törös analysed the results and all authors were involved in the discussion of the results. Bianca Törös drafted the paper and Sara Thulin Hedberg, Susanne Jacobsson, Hans Fredlund, Per Olcén and Paula Mölling revised the paper.

\section{References}

1. Cartwright KA, Stuart JM, Jones DM, Noah ND. The Stonehouse survey: nasopharyngeal carriage of meningococci and Neisseria lactamica. Epidemiol Infect. 1987;99(3):591-601. http://dx.doi.org/10.1017/S0950268800066449

2. Caugant DA. Population genetics and molecular epidemiology of Neisseria meningitidis. APMIS. 1998;106(5):505-25. http:// dx.doi.org/10.1111/j.1699-0463.1998.tb01379.x

3. Maiden MC, Bygraves JA, Feil E, Morelli G, Russell JE, Urwin $\mathrm{R}$, et al. Multilocus sequence typing: a portable approach to the identification of clones within populations of pathogenic microorganisms. Proc Natl Acad Sci USA. 1998;95(6):3140-5. http://dx.doi.org/10.1073/pnas.95.6.3140

4. Pace D, Pollard AJ. Meningococcal A. C, Y and W-135 polysaccharide-protein conjugate vaccines. Arch Dis Child. 2007;92(10):909-15. http://dx.doi.org/10.1136/adc.2006.111500

5. Stephens DS, Greenwood B, Brandtzaeg P. Epidemic meningitis, meningococcaemia, and Neisseria meningitidis. Lancet. 2007;369(9580):2196-210. http://dx.doi.org/10.1016/ S0140-6736(07)61016-2

6. Harrison LH, Trotter CL, Ramsay ME. Global epidemiology of meningococcal disease. Vaccine. 2009;27(Suppl 2):B51-63. http://dx.doi.org/10.1016/j.vaccine.2009.04.063

7. Hedberg ST, Törös B, Fredlund H, Olcén P, Mölling P. Genetic characterisation of the emerging invasive Neisseria meningitidis serogroup $Y$ in Sweden, 2000 to 2010. Euro Surveill. 2011;16(23):pii=19885.

8. Bröker M, Jacobsson S, DeTora L, Pace D, Taha MK. Increase of meningococcal serogroup $Y$ cases in Europe: a reason for concern? Hum Vaccin Immunother. 2012;8(5):685-8. http:// dx.doi.org/10.4161/hv.20098
9. Bröker M, Jacobsson S, Kuusi M, Pace D, Simões MJ, Skoczynska A, et al. Meningococcal serogroup Y emergence in Europe: Update 2011. Hum Vaccin Immunother. 2012;8(12). http://dx.doi.org/10.4161/hv.21794

10. Jolley K. Meningococcal typing. [Accessed 5 November 2012]. Available from: http://neisseria.org/nm/typing/

11. Jolley KA, Brehony C, Maiden MC. Molecular typing of meningococci: recommendations for target choice and nomenclature. FEMS Microbiol Rev. 2007;31(1):89-96. http:// dx.doi.org/10.1111/j.1574-6976.2006.00057.x

12. Taha MK, Vazquez JA, Hong E, Bennett DE, Bertrand S, Bukovski S, et al. Target gene sequencing to characterize the penicillin G susceptibility of Neisseria meningitidis. Antimicrob Agents Chemother. 2007;51(8):2784-92. http://dx.doi. org/10.1128/AAC.00412-07

13. Beernink PT, Granoff DM. The modular architecture of meningococcal factor $\mathrm{H}$-binding protein. Microbiology. 2009;155(Pt 9):2873-83. http://dx.doi.org/10.1099/ mic.0.029876-0

14. Pajon R, Beernink PT, Harrison LH, Granoff DM. Frequency of factor $\mathrm{H}$-binding protein modular groups and susceptibility to cross-reactive bactericidal activity in invasive meningococcal isolates. Vaccine. 2010;28(9):2122-9. http://dx.doi. org/10.1016/j.vaccine.2009.12.027

15. Yazdankhah SP, Lindstedt BA, Caugant DA. Use of variablenumber tandem repeats to examine genetic diversity of Neisseria meningitidis. J Clin Microbiol. 2005;43(4):1699-705. http://dx.doi.org/10.1128/JCM.43.4.1699-1705.2005

16. Yazdankhah SP, Lindstedt BA. Variable number tandem repeat typing of bacteria. Methods Mol Biol. 2007;396:395-405. http://dx.doi.org/10.1007/978-1-59745-515-2_25

17. Lindstedt BA. Multiple-locus variable number tandem repeats analysis for genetic fingerprinting of pathogenic bacteria. Electrophoresis. 2005;26(13):2567-82. http://dx.doi. org/10.1002/elps.200500096

18. Schouls LM, van der Ende A, Damen M, van de Pol I. Multiplelocus variable-number tandem repeat analysis of Neisseria meningitidis yields groupings similar to those obtained by multilocus sequence typing. J Clin Microbiol. 2006;44(4):150918. http://dx.doi.org/10.1128/JCM.44.4.1509-1518.2006

19. Törös B, Hedberg ST, Jacobsson S, Fredlund H, Olcén P, Mölling P. Evaluation of molecular typing methods for identification of outbreak-associated Neisseria meningitidis isolates. APMIS. 2013;121(6):503-10. http://dx.doi.org/10.1111/apm.12022

20. European Commission. Commission Decision of 28 April 2008 amending Decision 2002/253/EC laying down case definitions for reporting communical diseases to the Community network under Decision No 2119/98/EC of the European Parliament and of the Council. Official Journal of the European Union. Luxembourg: Publications Office of the European Union. 18.6.2008:L159/46. Available from: http://eur-lex.europa.eu/ LexUriServ/LexUriServ.do?uri=0J:L:2008:159:0046:0090:EN: PDF

21. Olcén $P$, Danielsson D, Kjellander J. The use of protein A-containing staphylococci sensitized with anti-meningococcal antibodies for grouping Neisseria meningitidis and demonstration of meningococcal antigen in cerebrospinal fluid. Acta Pathol Microbiol Scand B. 1975;83(4):387-96.

22. Mölling P, Jacobsson S, Bäckman A, Olcén P. Direct and rapid identification and genogrouping of meningococci and porA amplification by LightCycler PCR. J Clin Microbiol. 2002;40(12):4531-5. http://dx.doi.org/10.1128/ JCM.40.12.4531-4535.2002

23. Tettelin H, Saunders NJ, Heidelberg J, Jeffries AC, Nelson $\mathrm{KE}$, Eisen JA, et al. Complete genome sequence of Neisseria meningitidis serogroup B strain $M_{5} 8$. Science. 2000;287(5459):1809-15. http://dx.doi.org/10.1126/ science.287.5459.1809

24. Jolley K. Neisseria Multi Locus Sequence Typing website. [Accessed 5 November 2012]. Available from: http://pubmlst. org/neisseria/

25. Hunter PR, Gaston MA. Numerical index of the discriminatory ability of typing systems: an application of Simpson's index of diversity. J Clin Microbiol. 1988;26(11):2465-6.

26. Grundmann H, Hori S, Tanner G. Determining confidence intervals when measuring genetic diversity and the discriminatory abilities of typing methods for microorganisms. J Clin Microbiol. 2001;39(11):4190-2. http://dx.doi.org/10.1128/ JCM.39.11.4190-4192.2001

27. Rådström $P_{1}$, Fermér $C$, Kristiansen $B E$, Jenkins $A$, Sköld $O$, Swedberg G. Transformational exchanges in the dihydropteroate synthase gene of Neisseria meningitidis: a novel mechanism for acquisition of sulfonamide resistance. J Bacteriol. 1992;174(20):6386-93. 
28. Fiebelkorn KR, Crawford SA, Jorgensen JH. Mutations in folP associated with elevated sulfonamide MICs for Neisseria meningitidis clinical isolates from five continents. Antimicrob Agents Chemother. 2005;49(2):536-40. http://dx.doi. org/10.1128/AAC.49.2.536-540.2005

29. Elias J, Harmsen D, Claus H, Hellenbrand W, Frosch M, Vogel U. Spatiotemporal analysis of invasive meningococcal disease, Germany. Emerg Infect Dis. 2006;12(11):1689-95. http://dx.doi. org/10.3201/eid1211.060682

30. Lucidarme J, Newbold LS, Findlow J, Gilchrist S, Gray SJ, Carr AD, et al. Molecular targets in meningococci: efficient routine characterization and optimal outbreak investigation in conjunction with routine surveillance of the meningococcal group $B$ vaccine candidate, $f H B P$. Clin Vaccine Immunol.

2011;18(2):194-202. http://dx.doi.org/10.1128/CVI.00401-10

31. Elias J, Kritz P, Musilek M, Claus H, Frosch M, Vogel U.

Diversity of Multiple-Locus-VNTR-Analysis-(MLVA)-types in meningococcal strains from different epidemiological settings. Oral presentation 059, given at: 18th International Pathogenic Neisseria Conference; 2012 Sep 9-14; Würzburg, Germany; $p$ 133. 